\title{
Studies of a Lower-Hybrid Wave Driven Plasma Equilibrium with a Hybrid-MHD Model on the TST-2 Spherical Tokamak*)
}

\author{
Naoto TSUJII, Yusuke YOSHIDA, Yuichi TAKASE, Akira EJIRI, Osamu WATANABE, \\ Hibiki YAMAZAKI, Yi PENG, Kotaro IWASAKI, Yuki AOI, Yongtae KO, Kyohei MATSUZAKI, \\ James H.P. RICE and Yuki OSAWA \\ The University of Tokyo, Kashiwa 277-8561, Japan
}

(Received 29 November 2019 / Accepted 22 February 2020)

\begin{abstract}
Removal of the central solenoid is considered essential to realize a spherical tokamak fusion reactor, but non-inductive plasma start-up is a challenge. Start-up using lower-hybrid (LH) waves has been studied on the TST-2 spherical tokamak at the University of Tokyo. The equilibrium poloidal field is believed to be generated mostly by the wave driven fast electrons, which are highly non-thermal and have large orbit excursions from the flux surfaces due to low plasma current. Such an equilibrium can be qualitatively different from the GradShafranov equilibrium routinely used for internal magnetic field reconstruction in a tokamak. In this work, the effect of fast electrons on the MHD equilibrium was investigated by considering the equilibrium solution of the hybrid-MHD model [Y. Todo and A. Bierwage, Plasma Fusion Res. 9, 3403068]. The fast electron distribution function was estimated using a LH current drive simulation based on ray-tracing and an orbit-averaged FokkerPlanck solver. The equilibrium solution of the hybrid-MHD model was successfully fitted to the magnetic and kinetic measurements. The resulting poloidal flux function was more skewed towards the outboard side when fast electrons were introduced, which was more consistent with the density profile measured by the Thomson scattering diagnostic.
\end{abstract}

(C) 2020 The Japan Society of Plasma Science and Nuclear Fusion Research

Keywords: non-inductive start-up, MHD, equilibrium reconstruction, fast electron, lower-hybrid current drive

DOI: $10.1585 /$ pfr. 15.2402010

\section{Introduction}

Removal of the central solenoid may help realize an economical spherical tokamak fusion reactor since the aspect ratio can be reduced to operate at higher $\beta$. However, establishment of a reliable non-inductive plasma start-up method remains a challenge. Start-up using radio frequency (RF) waves is one of the promising candidates. It has been demonstrated experimentally that start-ups using lower-hybrid waves (LHW) [1-3] and electron cyclotron/Bernstein waves (ECW/EBW) $[4,5]$ are possible.

Quantitative description of the non-inductive start-up experiments are essential to predict the feasibility of these start-up scenarios in future devices. Numerical analysis has been performed to explain the start-up results with LHW [3, 6] and ECW/EBW [4]. For LH start-up, current drive analysis was performed with a coupled ray-tracing and Fokker-Planck simulation and the dependencies on the plasma parameters were qualitatively explained [3]. On the other hand, the predicted plasma current was substantially greater than what was measured experimentally. A time dependent hybrid-MHD simulation code MEGA [7] was used to find an equilibrium consistent with the LH driven fast electron distribution function [6]. The fast electron

author'se-mail: tsujii@k.u-tokyo.ac.jp

*) This article is based on the presentation at the 28th International Toki Conference on Plasma and Fusion Research (ITC28). current of the converged solution was much closer to the experimentally observed plasma current. The study also showed that the LH wave propagation and damping can be affected strongly by the internal current profile. Since internal current profile of the previous studies were reconstructed based only on the peripheral magnetics, the predictions had large uncertainty, and quantitative agreement between the simulation and the experiment had been poor.

Although the study with the MEGA code [6] showed that coupling of MHD and LH current drive simulation was the right direction to be pursued, the simulation, especially the MHD part, was computationally very expensive and not suitable for day-to-day analysis of the experiment. In this study, we considered a simpler problem of finding a best fit of the steady-state solution of the hybrid-MHD model to the experimental measurements. The difference between the best fit solutions of the hybrid-MHD model and the standard Grad-Shafranov equation was studied in detail.

\section{The Hybrid-MHD Model}

We have used a hybrid model of MHD and driftkinetic fast particles [7], which are fast electrons in this study. The modified Grad-Shafranov equation including the fast particle current [8] is derived below for reader's 
convenience. The divergence free magnetic field can be written

$$
\mathbf{B}=B_{\phi} \mathbf{e}_{\phi}+\frac{\nabla \psi}{R} \times \mathbf{e}_{\phi},
$$

in an axisymmetric system. $\psi$ is the poloidal flux per radian. Similarly, since the current is divergence free in equilibrium,

$$
\mu_{0} \mathbf{j}=\mu_{0} j_{\phi} \mathbf{e}_{\phi}+\frac{\nabla H}{R} \times \mathbf{e}_{\phi}
$$

$\mu_{0} H$ is the integrated poloidal current per radian. From Ampere's law,

$$
\begin{aligned}
& \mu_{0} j_{\phi}=-\frac{\Delta^{*} \psi}{R}, \\
& H=R B_{\phi},
\end{aligned}
$$

where

$$
\Delta^{*}=R \frac{\partial}{\partial R} \frac{1}{R} \frac{\partial}{\partial R}+\frac{\partial^{2}}{\partial Z^{2}} .
$$

The current $\mathbf{j}$ is decomposed into the bulk MHD fluid current $\mathbf{j}_{\mathrm{b}}$ and fast particle current $\mathbf{j}_{\mathrm{f}}$

$$
\begin{aligned}
& \mu_{0} \mathbf{j}=\mu_{0} \mathbf{j}_{\mathrm{b}}+\mu_{0} \mathbf{j}_{\mathrm{f}}, \\
& \mu_{0} \mathbf{j}_{\mathrm{b}}=\mu_{0} j_{\mathrm{b} \phi} \mathbf{e}_{\phi}+\frac{\nabla F}{R} \times \mathbf{e}_{\phi}, \\
& \mu_{0} \mathbf{j}_{\mathrm{f}}=\mu_{0} j_{\mathrm{f} \phi} \mathbf{e}_{\phi}+\frac{\nabla G}{R} \times \mathbf{e}_{\phi} .
\end{aligned}
$$

$F$ and $G$ are the integrated poloidal currents for the bulk and fast particles, respectively. Note $H=F+G$. Assuming static (no flow) equilibrium,

$$
\mathbf{j}_{\mathrm{b}} \times \mathbf{B}=\nabla P .
$$

$P$ is the bulk pressure. Substituting Eqs. (1)-(8),

$$
-\frac{\Delta^{*} \psi}{R}=\mu_{0} R \frac{\mathrm{d} P}{\mathrm{~d} \psi}+\frac{H}{R} \frac{\mathrm{d} F}{\mathrm{~d} \psi}+\mu_{0} j_{\mathrm{f} \phi} .
$$

This equation is almost the same as the standard GradShafranov equation except that there are two additional terms: the toroidal current density $j_{\mathrm{f} \phi}$ and the integrated poloidal current $G$ of the fast particles. By dotting Eq. (9) with $\mathbf{B}$ and $\mathbf{j}_{\mathrm{b}}$, it can be shown that the bulk pressure $P$ and the bulk poloidal current $F$ are still flux functions. On the other hand, $j_{\mathrm{f} \phi}$ and $G$ are not flux functions in general, and the equilibrium with fast particles can be qualitatively different from what can be described by the original GradShafranov equation.

The fast particle current density $\mathbf{j}_{\mathrm{f}}$ can be obtained by integrating the distribution function of the fast particles. In this study, we used the drift-kinetic picture. Since the fast electrons in the tens of $\mathrm{keV}$ range can be considered collisionless (time for electrons to transit a full orbit is shorter compared to the collision time), the equilibrium fast particle distribution function $f$ were considered to be a function of the constants of motion

$$
f=f\left(E, \mu, \psi^{*}, \sigma\right),
$$

where

$$
\begin{aligned}
& E=\frac{m_{\mathrm{e}}}{2}\left(v_{\|}^{2}+v_{\perp}^{2}\right), \\
& \mu=\frac{m_{\mathrm{e}} v_{\perp}^{2}}{2 B} \\
& \psi^{*}=\psi+\frac{H}{\Omega_{\mathrm{e}}} v_{\|}, \\
& \sigma=v_{\|} /\left|v_{\|}\right| .
\end{aligned}
$$

Here, $v_{\|}$and $v_{\perp}$ are the velocities of the particle parallel and perpendicular to the magnetic field, respectively, $E$ is the energy, $\mu$ is the magnetic moment, $\psi^{*}$ is the toroidal angular momentum (per charge) averaged over the gyroperiod, and $\sigma$ is the parallel velocity sign. $\Omega_{\mathrm{e}}=-e B / m_{\mathrm{e}}$ is the algebraic electron cyclotron (angular) frequency. To first order in gyro-radius, the current of the drift-kinetic distribution function can be written,

$$
\mathbf{j}_{\mathrm{f}}=j_{\mathrm{f} \|} \mathbf{b}+\frac{P_{\mathrm{f} \|}-P_{\mathrm{f} \perp}}{B} \nabla \times \mathbf{b}+\frac{\mathbf{b} \times \nabla P_{\perp}}{B},
$$

where

$$
\begin{aligned}
& j_{\mathrm{f} \|}=-e \int d^{3} v f \\
& P_{\mathrm{f} \|}=m_{\mathrm{e}} \int d^{3} v v_{\|}^{2} f, \\
& P_{\mathrm{f} \perp}=m_{\mathrm{e}} \int d^{3} v v_{\perp}^{2} f .
\end{aligned}
$$

For the best accuracy, the fast particle distribution function $f\left(E, \mu, \psi^{*}, \sigma\right)$ should be obtained directly from the coupled ray-tracing and orbit-averaged Fokker-Planck simulation. However, in this work, we used an analytic distribution function model to see first if a plausibly looking fast electron distribution function can modify the standard GradShafranov equilibrium in any qualitative way.

\section{Experimental Setup}

TST-2 is a spherical tokamak $\left(R_{0}=0.36 \mathrm{~m}, a=\right.$ $0.23 \mathrm{~m}, B_{\mathrm{t} 0}=0.3 \mathrm{~T}, I_{\mathrm{p}}<0.14 \mathrm{MA}$ ) [9] located at the University of Tokyo. The magnets are powered by capacitor banks and the on-axis toroidal field is limited to $0.16 \mathrm{~T}$ for LH start-up discharges due to longer pulse length. The maximum plasma current achieved for LH start-up discharges is $\sim 25 \mathrm{kA}$ which is about a quarter of that of the typical discharges driven by the central solenoid.

Four $100 \mathrm{~kW}$ sources at $200 \mathrm{MHz}$ are available for LH current drive. Presently, two capacitively-coupled combline (CCC) antennas are installed to excite LH waves $[1,2]$. The outboard midplane antenna launches the waves at $n_{\|}=6.0$ and has $200 \mathrm{~kW}$ source power. The top antenna launches the waves at $n_{\|}=4.7$ at the center of the antenna, and has $100 \mathrm{~kW}$ source power. High $n_{\|}$is needed in TST-2 due to low magnetic field strength. Absorption on the bulk electrons occurs at $n_{\|}>20$.

A $5 \mathrm{~kW}$ electron cyclotron heating $(\mathrm{ECH})$ system at $2.45 \mathrm{GHz}$ is also available for preionization of the plasma. 
ECH was used only to assist breakdown, and turned off during the plasma current ramp-up for the results shown in this paper.

The electron profiles were measured using a tangential Thomson scattering diagnostic [10,11]. Measuring profiles for LH driven plasmas had been extremely challenging due to the low density $\left(\sim 10^{17} \mathrm{~m}^{-3}\right)$, but now we are able to obtain profile measurements routinely by accumulating signals for 3 - 5 shots. The measurement points were chosen to cover both the high-field side and low-field side of the magnetic axis. This arrangement turned out to be critical for this work since the measured profile contained information of the internal poloidal flux function.

\section{LH Start-Up Experimental Results}

The time traces of the typical fully non-inductive $\mathrm{LH}$ start-up discharge are shown in Fig. 1. The loop voltage is slightly negative due to the back EMF of the ramping plasma current. The flat top plasma current was sustained using only the top-launch antenna for simpler analysis. The outboard antenna was used for fast start-up of the plasma current since the top-launch antenna was less effective at the very early stage of the start-up due to its reliance on the up-shift of the parallel refractive index [3]. The electron profiles measured by the Thomson scattering diagnostic are shown in Fig. 2. The density profile was peaked, indicating finite confinement and current density up to the magnetic axis. The temperature profiles were hollow, which suggests that the heating, and hence, current drive occurred near the edge of the plasma.

\section{Hybrid-MHD Equilibrium Fitting \\ 5.1 Ray-tracing and Fokker-Planck analysis of the $L H$ current drive}

To get a rough picture of the fast electron distribution function, LH current drive simulation was performed using a ray-tracing code GENRAY [12] and an orbit-averaged Fokker-Planck code CQL3D [13]. The two codes are coupled to simulate self-consistent wave damping and a fast electron distribution function. Both codes assumed zero orbit width in this work.

The ray trajectories of the waves launched from the top antenna is shown in Fig. 3. The same ray trajectories in phase space are shown in Fig. 4. The red hatched area on the low velocity side shows the region below three times the electron thermal velocity. The rays propagating into this regime quickly get absorbed completely by the bulk electrons. The black hatched area on the high velocity side shows the velocity above the mode conversion condition at the toroidal field strength on the high-field side of the flux surface. LH waves convert to fast waves at this boundary and can not propagate into this region. The rays propagating above the midplane experience strong up-shift of the parallel refractive index $n_{\|}$(down-shift of the parallel phase velocity) due to the $1 / R$ gradient of the toroidal

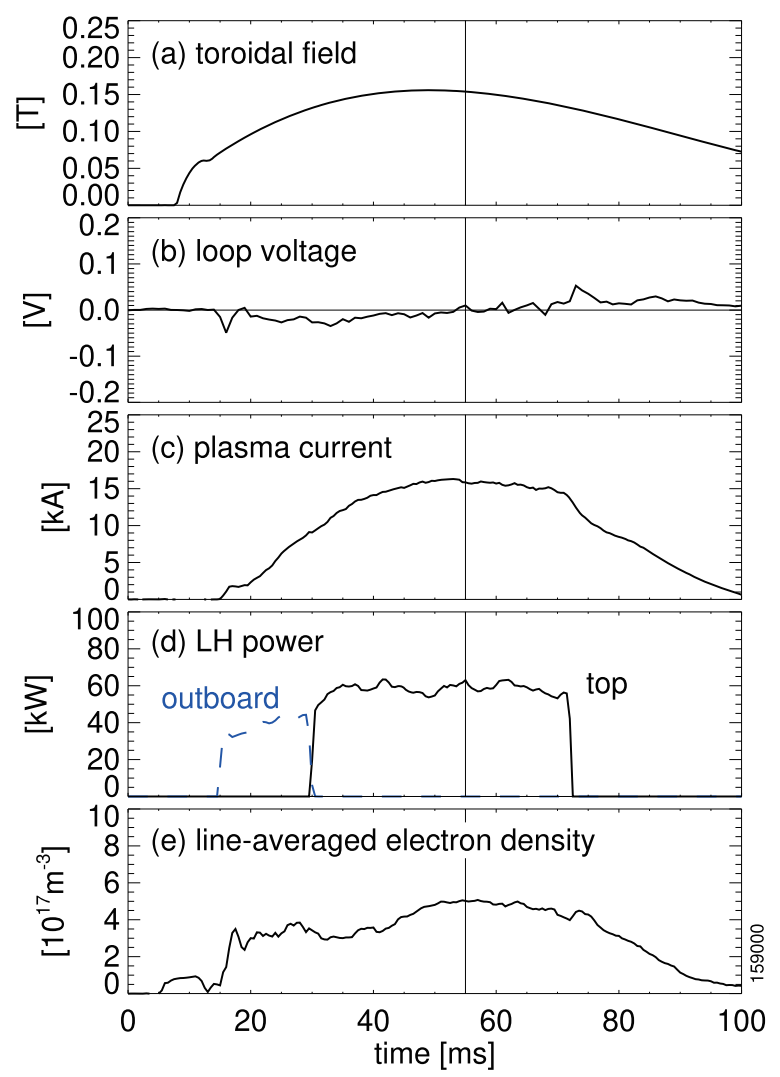

Fig. 1 The time traces of (a) toroidal field (b) loop voltage (c) plasma current (d) net LH power of the top-launch antenna (black solid curve) and the outboard-launch antenna (blue dashed curve) (e) line-averaged electron density. The vertical line shows $55 \mathrm{~ms}$ when the Thomson scattering measurement was performed.

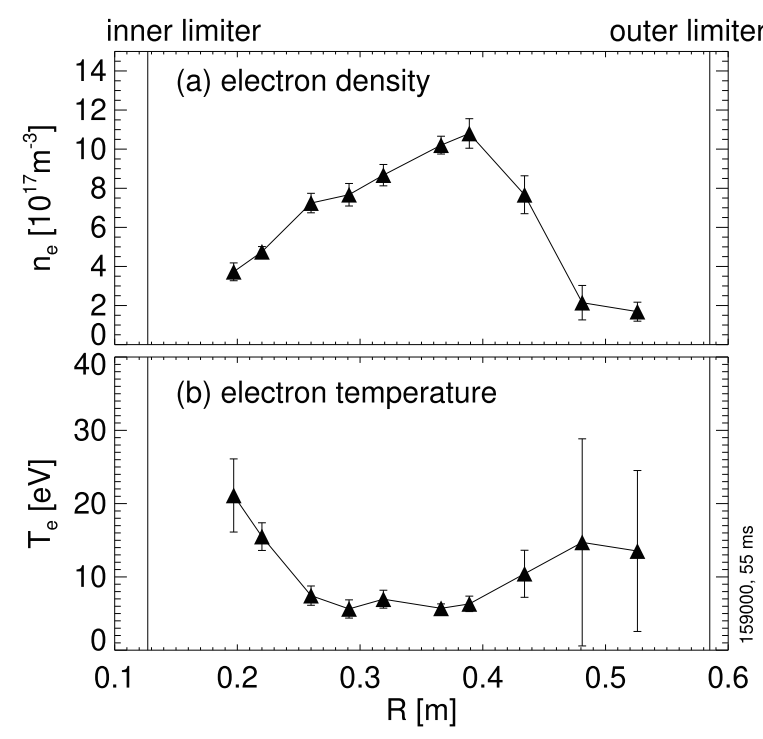

Fig. 2 The electron (a) density and (b) temperature profiles at $55 \mathrm{~ms}$ for the discharge shown in Fig. 1. The inner and outer limiter locations are also shown with vertical lines.

field (Fig. 4). This up-shift in $n_{\|}$results in strong wave diffraction which turns the rays radially outward. Since the rays are reflected back in at the low density cutoff, they 

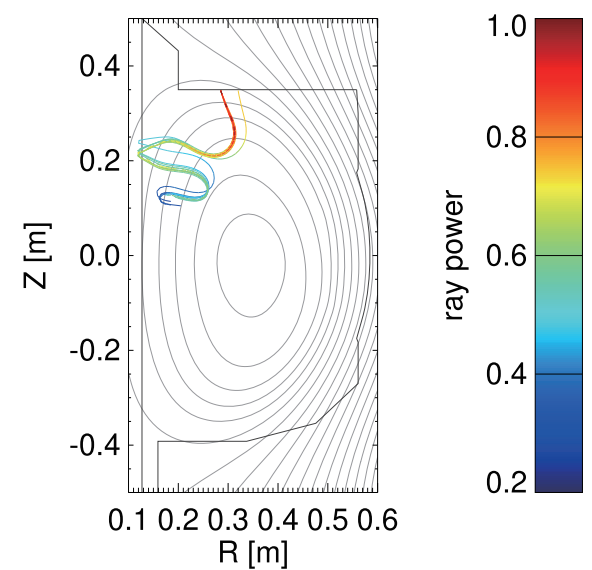

Fig. 3 The ray trajectories of the waves launched from the top antenna. The color shows the power contained in each ray (normalized to the maximum power).
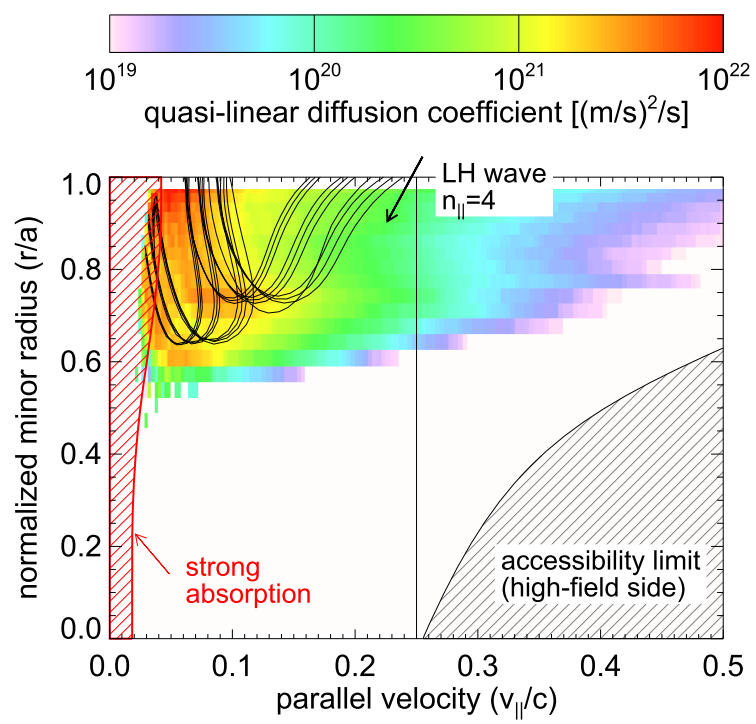

Fig. 4 The phase space ray trajectories of the waves launched from the top antenna. The color shows the strength of the quasilinear diffusion coefficient. The red hatched area shows the absorption limit and the black hatched area shows the mode conversion limit. The vertical line shows the parallel velocity corresponding to $n_{\|}=4$.

travel back and forth between $0.6<r / a<1.0$ while continuously slowing down in parallel velocity until they are absorbed completely by the bulk electrons. By launching waves at a relatively low $n_{\|}$, and letting them up-shift as they propagate, broad $n_{\|}$spectrum ideal for efficient current drive could be generated inside the plasma.

\subsection{The fast electron distribution function model}

It can be seen in Fig. 4 that strong quasilinear diffusion is localized at the plasma edge and extends up to about $n_{\|}=4$ in velocity space. Based on this observation, an analytic model of the fast electron distribution function
$f\left(E, \mu, \psi^{*}, \sigma\right)$ was formulated as

$$
\begin{aligned}
f\left(E, \mu, \psi^{*}, \sigma\right)= & \mathcal{N} \exp \left(-\frac{\mu B_{0}}{T_{\mathrm{e} 0}}\right) \exp \left(-\frac{\left(\psi^{*}-\psi_{0}\right)^{2}}{\Delta \psi^{2}}\right) \\
& \text { if } E_{\min }<E<E_{\max }, \sigma=\sigma_{0},
\end{aligned}
$$

and $f\left(E, \mu, \psi^{*}, \sigma\right)=0$ otherwise. The parameters $B_{0}, \psi_{0}$, $\Delta \psi$ are calculated during each fitting iteration as

$$
\begin{aligned}
& B_{0}=B\left(R_{\mathrm{LH}}, Z=0\right), \\
& \psi_{0}=\psi\left(R_{\mathrm{LH}}, Z=0\right), \\
& \Delta \psi=w_{\mathrm{LH}} \frac{\partial \psi}{\partial R}\left(R_{\mathrm{LH}}, Z=0\right) .
\end{aligned}
$$

The normalization of the distribution function $\mathcal{N}$ is also recalculated each iteration to fix the total current carried by the fast electrons

$$
I_{\mathrm{f}}=\int \mathrm{d} R \mathrm{~d} Z j_{\mathrm{f} \phi} .
$$

The input parameters are the radial position $R_{\mathrm{LH}}$ and width $w_{\mathrm{LH}}$ of the current, minimum and maximum energy of the fast electron plateau $E_{\min }, E_{\max }$, temperature $T_{\mathrm{e} 0}$ and total current $I_{\mathrm{f}}$.

\subsection{Determination of the model function pa- rameters}

The model function parameters were determined to best describe the equilibrium at $55 \mathrm{~ms}$ of the discharge shown in Fig. 1. The plasma current was in the direction of the toroidal field $\left(\sigma_{0}=-1\right)$ for this discharge.

Since the minimum velocity of the fast electron plateau is known to be around $\sim 3$ times the electron thermal velocity, the minimum energy was set to

$$
E_{\min }=9 T_{\mathrm{e} 0}
$$

and $T_{\mathrm{e} 0}=20 \mathrm{eV}$ according to the measurement shown in Fig. 2. The maximum energy corresponds to the maximum phase velocity of the LH waves.

$$
E_{\max }=\frac{1}{2} m_{\mathrm{e}}\left(\frac{c}{n_{\|}}\right)^{2} .
$$

From Fig. 4 , we set $n_{\|}=4$. Because the plasma diffraction only slows down the rays, the maximum phase velocity is determined largely by the launched $n_{\|}$, and we expect this number does not depend strongly on the magnetic geometry or the plasma conditions.

The simulated radial distribution of the quasi-linear diffusion coefficient (Fig. 4) is likely to be inaccurate since the fast electrons are assumed to have zero orbit width. Because we did not have a means to obtain a quantitatively accurate radial distribution, we performed a preliminary scan of the radial distribution parameters $\left(R_{\mathrm{LH}}\right.$ and $\left.w_{\mathrm{LH}}\right)$ with reduced degrees of freedom $\left(P_{2}\right.$ set to zero in Eq. (27)). The $\chi^{2}$ for magnetics fitting is plotted versus 


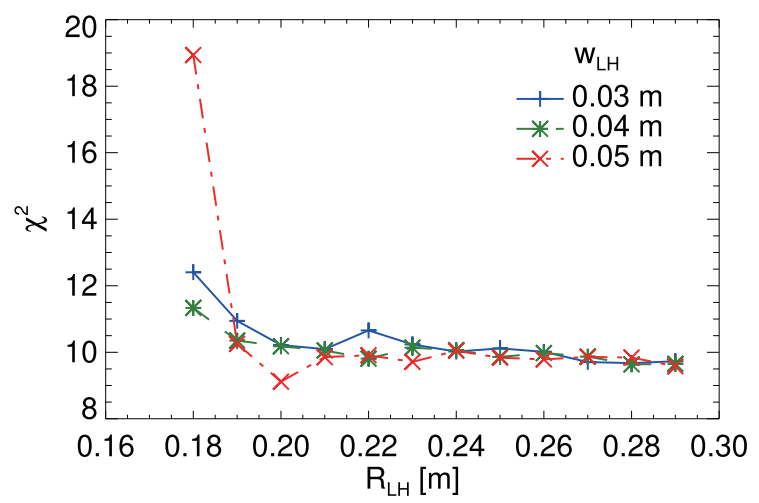

Fig. 5 The $\chi^{2}$ of the fitted solution plotted versus $R_{\mathrm{LH}}, 70 \%$ fast electron current fraction. Blue plus symbols: $w_{\mathrm{LH}}=$ $0.03 \mathrm{~m}$, green asterisks: $w_{\mathrm{LH}}=0.04 \mathrm{~m}$, red crosses: $w_{\mathrm{LH}}=0.05 \mathrm{~m}$.

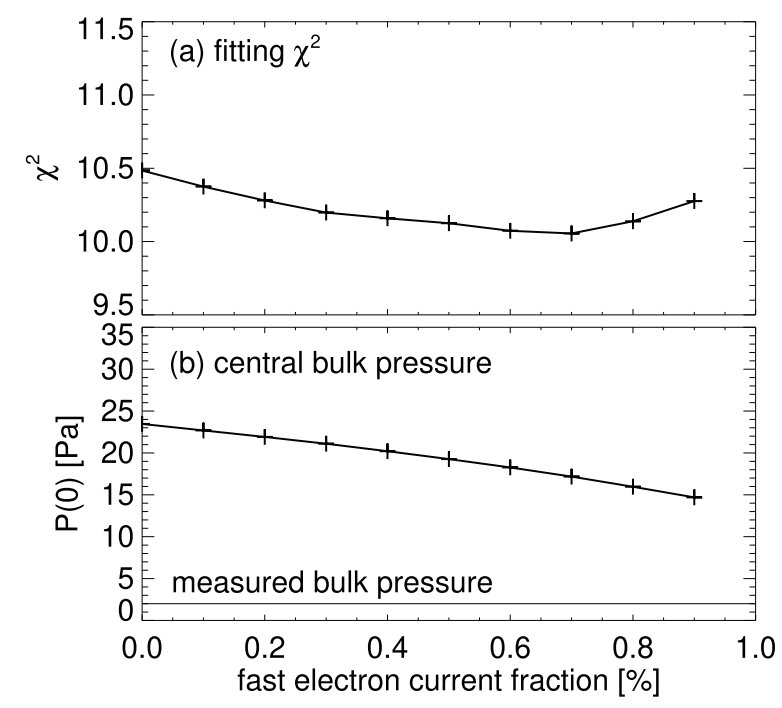

Fig. 6 (a) Fitting $\chi^{2}$ and (b) central bulk pressure $P(0)$ versus the fast electron current fraction. The bulk pressure estimation from the Thomson scattering measurement is also shown with the horizontal line in (b).

the radial distribution parameters in Fig. 5. The fast electron current fraction was $70 \%$. The fitting $\chi^{2}$ did not vary much for a wide range of $R_{\mathrm{LH}}$ and $w_{\mathrm{LH}}$. This is likely due to lack of information of the internal current profile. To see the effect of localized current source, we picked a value of width which is small but still compatible with the mesh size $(0.025 \mathrm{~m}): w_{\mathrm{LH}}=0.04 \mathrm{~m}$. For the radial location, we picked $R_{\mathrm{LH}}=0.21 \mathrm{~m}$ to reflect our expectation that the current drive was probably far off-axis, but also where $\chi^{2}$ was not too large.

The fraction of the fast electron current to the total plasma current was scanned and the resulting fitting $\chi^{2}$ is plotted in Fig. 6 for the above determined parameters. The fitting remained stable up to $90 \%$ fast electron current fraction with small increase in $\chi^{2}$ at the highest value of the current fraction $(>70 \%)$. The fitted central bulk pressure decreased steadily as the bulk current was replaced by the fast electron current towards higher current fraction. With the present modeling, the minimum central bulk pressure was $15 \mathrm{~Pa}$ at $90 \%$ fast electron current fraction, whereas the estimation from the Thomson scattering measurement was at most $2 \mathrm{~Pa}$. We believe the discrepancy is due to the inconsistency between the electron distribution function model and the actual fast electron distribution in the experiment. In other words, some of the fast electron current that could not be described by the present distribution function model was being carried by the "bulk" pressure and "bulk" poloidal current that were fitted as free functions. It is not clear how much improvement can be made when a realistic distribution function from a numerical simulation is used. For the following analysis, fast electron current fraction of $70 \%$ was used so that the equilibrium field was dominated by the fast electron current while still ensuring stable convergence and good fitting.

\subsection{Fitting results}

The two bulk free functions were parameterized as

$$
\begin{aligned}
& P^{\prime}(x)=\left(1-P_{2}-P_{0}\right) x^{3}+P_{2} x^{2}+P_{0} \\
& F F^{\prime}(x)=\left(1-F_{2}-F_{0}\right) x^{3}+F_{2} x^{2}+F_{0}
\end{aligned}
$$

where $x=\left(\psi-\psi_{\text {axis }}\right) /\left(\psi_{\mathrm{LCFS}}-\psi_{\text {axis }}\right)$ is the normalized poloidal flux and $\psi_{\text {axis }}$ and $\psi_{\text {LCFS }}$ are the values of the poloidal flux $\psi$ at the magnetic axis and the last closed flux surface (LCFS), respectively. Note that $P^{\prime \prime}(0)=0$ (flat current profile around the axis) and $P^{\prime}(1)=0$ (current goes to zero at the LCFS).

The magnetic axis needed to be constrained for stable fitting with the above flexibility of the bulk free functions. The fitting $\chi^{2}$ is plotted versus the major radius of the magnetic axis in Fig. 7. With only the peripheral magnetics (Fig. 7 (a)), $\chi^{2}$ varied little for a wide range of magnetic axis positions, with or without the fast electrons. Since our Thomson scattering system can capture the full radial density profile, this can be used as a constraint for the internal magnetic field structure. Assuming that the bulk density profile is a flux function, the measured density profile was fitted as a polynomial function of the normalized poloidal flux $x$. The combined $\chi^{2}$ of this density fitting and magnetics fitting error is shown in Fig. 7 (b). The best fit solutions were obtained at magnetic axis positions of $0.361 \mathrm{~m}$ without fast electrons, and $0.363 \mathrm{~m}$ with fast electrons.

Figure 8 shows the best fit solution of the poloidal flux with fast electrons (red solid curves) and without fast electrons (black dashed curves). The edge magnetic structure did not vary much with or without the fast electrons, since it is constrained strongly by the peripheral magnetics. However, the inner flux surfaces did change when fast electrons were introduced. The bulk pressure $P$ and poloidal current $F$ functions of these solutions are shown in Fig. 9. Both the bulk pressure and the paramagnetic current decreased as they were replaced by the fast electron 

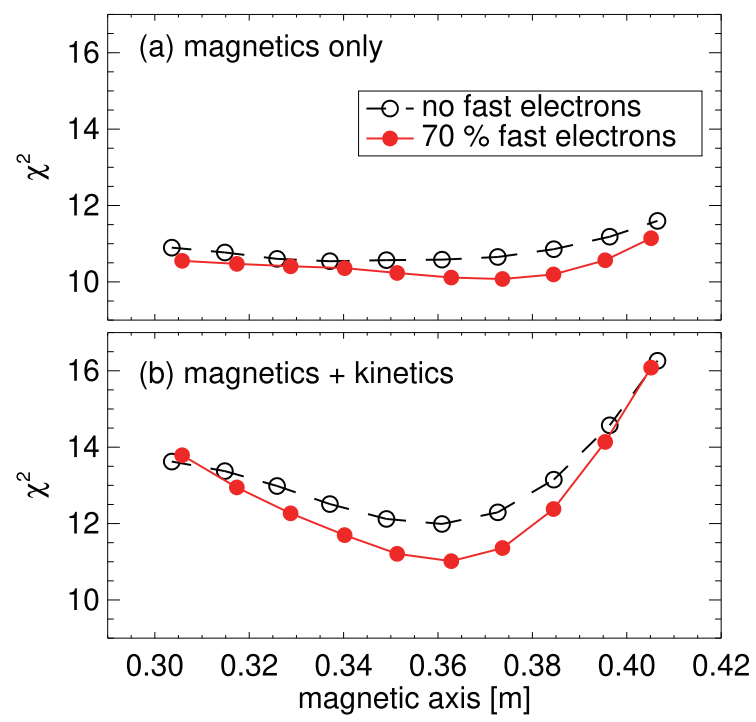

Fig. 7 Fitting $\chi^{2}$ plotted versus the major radius of the magnetic axis. (a) $\chi^{2}$ of magnetics only. (b) Combined $\chi^{2}$ of magnetics and kinetics. Open black circles: no fast electrons, filled red circles: $70 \%$ of the plasma current carried by the fast electrons.

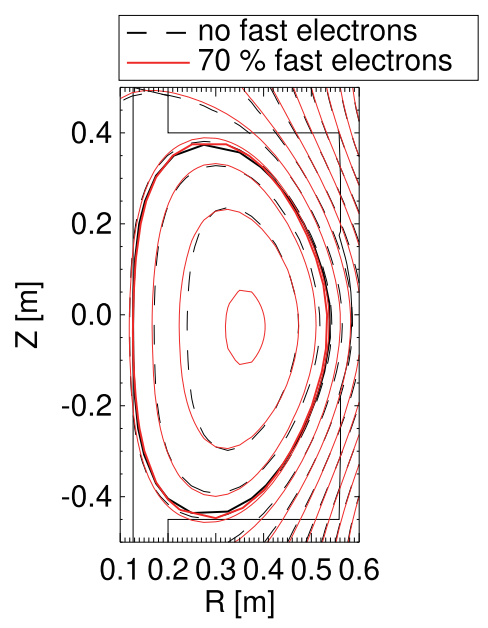

Fig. 8 The best fit poloidal flux solution without fast electrons (black dashed contours) and with $70 \%$ of the plasma current carried by fast electrons (red solid contours).

current. The bulk poloidal current became slightly diamagnetic in this case and canceled part of the fast electron current (shown later in Fig. 11 (b)). This may be because the fast electron current profile assumed here was too strongly peaked.

To see how the kinetic fitting (electron density profile fitting) improved, the measured electron density profile is plotted together with the fitted profiles in Fig. 10. Although the difference with and without fast electrons were not drastic, there was a systematic change when the fast electrons were introduced. Without fast electrons, the flux function (black dashed curve) was more or less symmetric in the radial direction. This resulted in poor fitting espe-

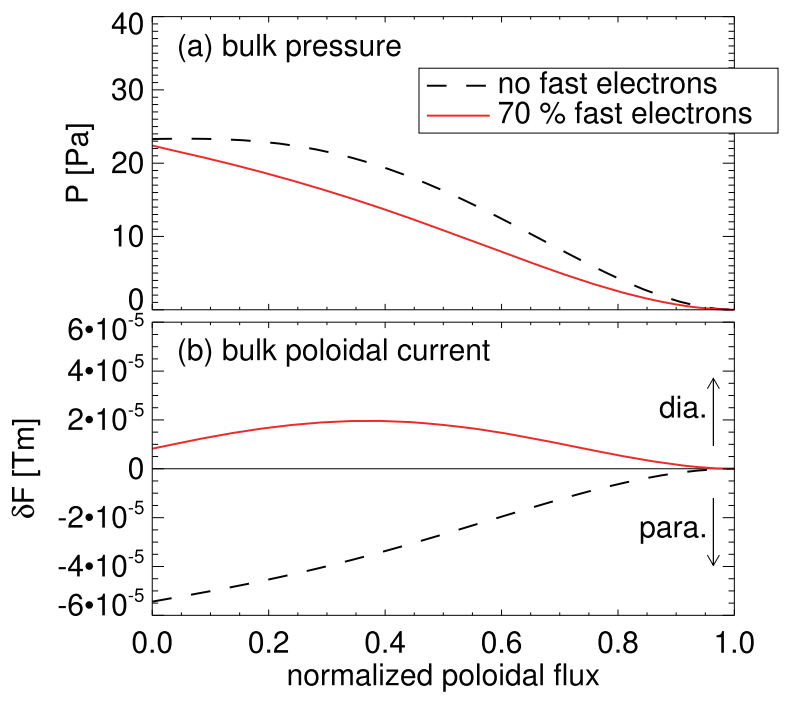

Fig. 9 The two bulk free functions of the best fit solution. (a) The bulk pressure function $P(x)$. (b) The bulk poloidal current function $F(x)$ with respect to the vacuum value $\left(\delta F=F-R_{0} B_{\mathrm{t} 0}\right) . \delta F>0$ for a diamagnetic plasma.

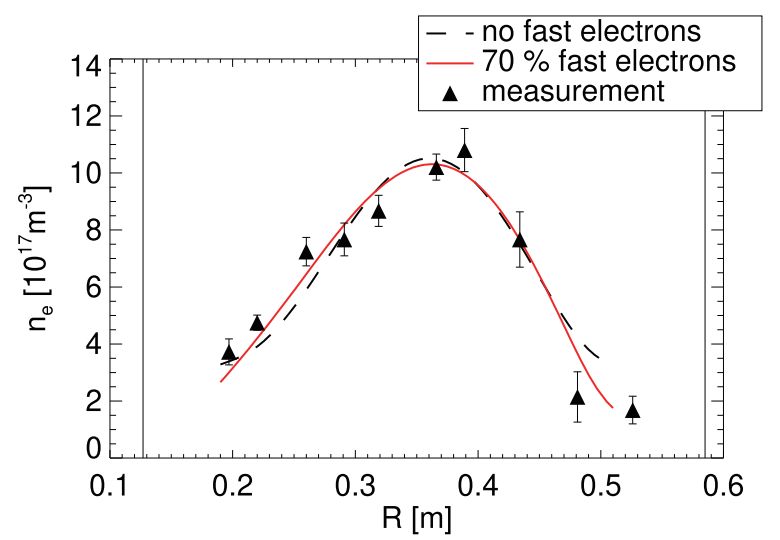

Fig. 10 The measured electron density profile (triangles with errorbars) and fitted curves without fast electrons (black dashed curve) and with $70 \%$ of the plasma current carried by fast electrons (red solid curve).

cially around $R \sim 0.5 \mathrm{~m}$. With fast electrons, however, the flux function was more skewed toward the low-field side, improving the fit at these points.

The change in the shape of the flux function can be understood from the radial current profile. The current profiles along the midplane are shown in Fig. 11. When fast electrons were included, the current density became much higher on the high-field side compared to the lowfield side. This is because the fast electrons have high parallel velocity and low perpendicular velocity, and do not slow down significantly on the high-field side compared to the low-field side. With similar parallel velocity on the high and low field sides, there is a simple $1 / R$ geometric increase in the current density on the high-field side. The result is a skewed poloidal flux function, which fits our 


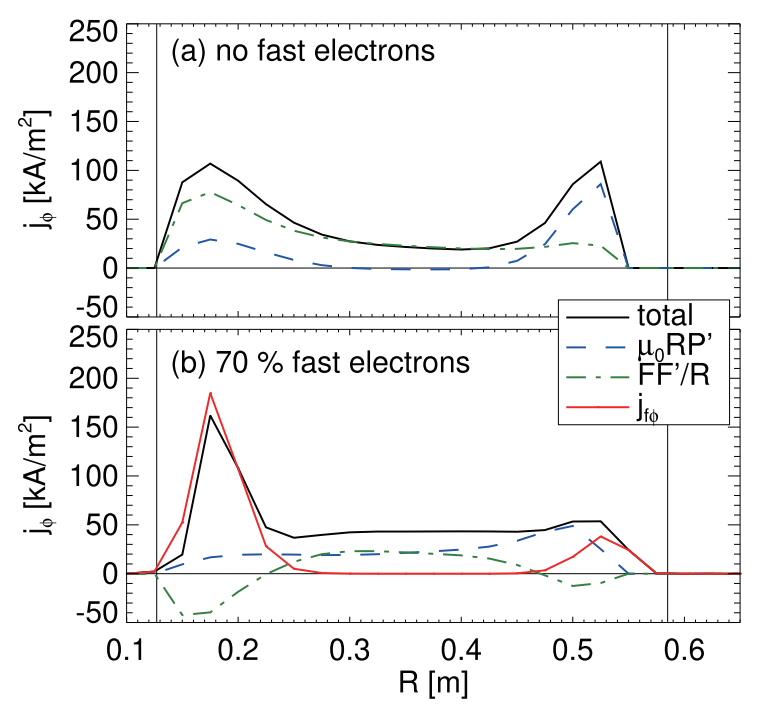

Fig. 11 The toroidal current density (a) without fast electrons and (b) with $70 \%$ of the plasma current carried by the fast electrons. Black solid curve: total current, blue dashed curve: pressure contribution, green dash-dotted curve: poloidal current contribution, red dash-threedotted curve: fast electron current.

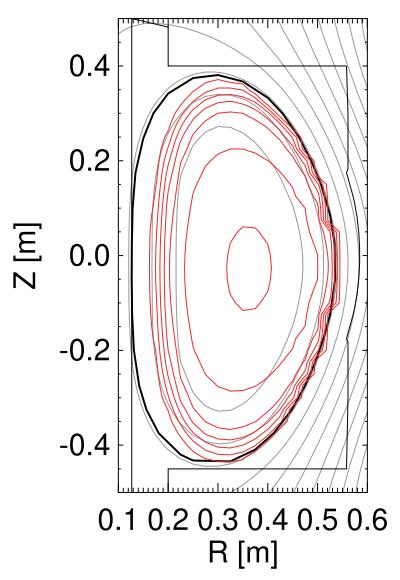

Fig. 12 The total (bulk and fast electron) poloidal current profile (red contours). The poloidal flux contours are shown in gray and the LCFS with black.

measured density profile better in this case.

For completeness, the contour plot of the integrated poloidal current $H$ is shown in Fig. 12. It can be seen that the poloidal current no longer flows along the flux surfaces when fast electrons are introduced.

\section{Discussion}

The fast electron current can be qualitatively different from the bulk current in two distinctive ways. The first difference is due to the excursion of the electron orbit from the flux surfaces (finite orbit effects). This can be seen clearly, for example, in Fig. 12. However, we do not know if this change really helped improve the accuracy of our equilibrium description at this point. The second difference, which we did not anticipate when we started this study, is due to the non-thermal nature of the fast electron distribution function. For our LH driven plasma, this second effect was actually substantial, resulting in a current profile much more concentrated on the high-field side which had previously been difficult to capture with the standard equilibrium fitting using the smooth model functions with small degrees of freedom. The modified current profile generated the poloidal field structure that was more consistent with the density profile measured by the Thomson scattering diagnostic, indicating that this is in fact a more accurate description of the system.

We expect that the majority of the plasma current is carried by the fast electrons in our LH driven plasmas simply because the fast electron velocities are more than 100 times higher than the thermal velocity of the bulk electrons. If this is the case, the current carried by the fast electrons should ultimately replace majority of the bulk current presently assumed in the equilibrium reconstruction. For the result shown in this paper, we could obtain an equilibrium up to $90 \%$ of the current carried by the fast electrons despite the crude analytic model of the fast electron distribution function, which is quite promising. It is unclear at this point how large a fast electron current fraction can be in this type of analysis. The analytic distribution function used in this paper is by no means the most accurate estimation, but chosen only for the sake of simplicity, since our purpose was to see precisely what the effect of fast particles were to the types of equilibrium we were interested in. Since now we see that this kind of model can actually improve our equilibrium description in a reasonable way, our next step is to use the wave and the Fokker-Planck simulations to obtain a realistic estimate of the distribution function and use them directly in the equilibrium reconstruction to further improve the accuracy.

\section{Conclusions and Future Work}

The effect of non-thermal fast electrons on the MHD equilibrium was investigated for a LH start-up plasma on the TST-2 spherical tokamak. Equilibrium fitting was performed with a steady-state solution of a hybrid-MHD model including toroidal and poloidal currents of the driftkinetic fast electrons. An analytic model of the fast electron distribution function was formulated which reflected the result of the current drive analysis by ray-tracing and Fokker-Planck simulations. A hybrid-MHD equilibrium with $70 \%$ of the current carried by the fast electrons could be found, which had better fitting $\chi^{2}$ than fitting to the standard Grad-Shafranov equilibrium. Most of the improvement in $\chi^{2}$ was from the better fit of the electron density profile which was assumed to be a flux function. The fast electron current was concentrated on the high-field side which generated poloidal flux profile skewed towards the low-field side, more consistently with the density profile measured by the Thomson scattering diagnostic. 
For future work, a finite orbit current drive analysis with ray-tracing and Fokker-Planck codes are necessary to couple their predictions directly to the hybrid-MHD equilibrium fitting constructed here. A more direct validation of the hybrid-MHD model is also needed with internal current profile diagnostics. A microwave polarimeter is being developed on TST-2 for this purpose.

\section{Acknowledgements}

This work was supported by JSPS KAKENHI Grant number 18K13524 and NIFS Collaboration Research Program NIFS18KOAR22 and NIFS12KUTR078.

[1] S. Yajima, Y. Takase, Y. Tajiri, Y. Takei, N. Tsujii, H. Yamazaki, C.P. Moeller and T. Shinya, Nucl. Fusion 59, 066004 (2019).

[2] T. Shinya, Y. Takase, S. Yajima, C. Moeller, H. Yamazaki, N. Tsujii, Y. Yoshida, A. Ejiri, H. Togashi, K. Toida, H. Furui, H. Homma, K. Nakamura, B. Roidl, M. Sonehara, W. Takahashi and T. Takeuchi, Nucl. Fusion 57, 036006 (2017).

[3] N. Tsujii, Y. Takase, A. Ejiri, T. Shinya, H. Togashi, S. Yajima, H. Yamazaki, C.P. Moeller, B. Roidl, M. Sonehara, W. Takahashi, K. Toida and Y. Yoshida, Nucl. Fusion 57, 126032 (2017).

[4] T. Maekawa, T. Yoshinaga, M. Uchida, F. Watanabe and H. Tanaka, Nucl. Fusion 52, 083008 (2012).

[5] A. Ejiri, Y. Takase, T. Oosako, T. Yamaguchi, Y. Adachi,
O. Watanabe, Y. Nagashima, B.I. An, H. Kobayashi, H. Kurashina, H. Hayashi, H. Matsuzawa, K. Yamada, H. Tojo, T. Masuda, M. Sasaki, R. Kumazawa, H. Kasahara and F. Shimpo, Nucl. Fusion 49, 065010 (2009).

[6] B. Roidl, Y. Todo, Y. Takase, N. Tsujii, A. Ejiri, Y. Yoshida, S. Yajima and T. Shinya, Comput. Phys. Commun. 230, 38 (2018).

[7] Y. Todo and A. Bierwage, Plasma Fusion Res. 9, 3403068 (2014).

[8] E.V. Belova, N.N. Gorelenkov and C.Z. Cheng, Phys. Plasmas 10, 3240 (2003).

[9] Y. Takase, A. Ejiri, N. Kasuya, T. Mashiko, S. Shiraiwa, L.M. Tozawa, T. Akiduki, H. Kasahara, Y. Nagashima, H. Nozato, H. Wada, H. Yamada, T. Yamada and K. Yamagishi, Nucl. Fusion 41, 1543 (2001).

[10] Y. Kawamata, A. Ejiri, K. Matsuzaki, Y. Takase, N. Tsujii, T. Onchi and Y. Nagashima, Plasma Fusion Res. 14, 1402072 (2019).

[11] H. Togashi, A. Ejiri, H. Homma, T. Shinya, Y. Takase, K. Toida, N. Tsujii, T. Yamaguchi, Y. Yoshida, H. Furui, M. Sonehara, K. Nakamura, W. Takahashi, T. Takeuchi, S. Yajima, H. Yamazaki, M. Hasegawa, Y. Nagashima and H. Tojo, J. Instrumentation 10, C12020 (2015).

[12] A.P. Smirnov and R.W. Harvey, Bull. Am. Phys. Soc. 39, 1626 (1994).

[13] R.W. Harvey and M.G. McCoy, in Proceedings of the IAEA Technical Committee Meeting on Simulation and Modeling of Thermonuclear Plasmas, Montreal, Canada, 1992 (USDOC NTIS Document No. DE93002962), page 40, 11 (1992). 